\title{
¿Seasonal Gap Theory for ENSO Phase Locking
}

\author{
SOONG-KI KIM ${ }^{\mathrm{a}}$ AND SOON-IL AN $\mathrm{AN}^{\mathrm{a}, \mathrm{b}}$ \\ ${ }^{a}$ Department of Atmospheric Sciences \& Irreversible Climate Change Research Center, Yonsei University, Seoul, South Korea \\ ${ }^{\mathrm{b}}$ Division of Environmental Science and Engineering, Pohang University of Science and Technology (POSTECH), \\ Pohang, South Korea
}

(Manuscript received 29 June 2020, in final form 30 January 2021)

\begin{abstract}
The life cycle of El Niño-Southern Oscillation (ENSO) typically follows a seasonal march, with onset in spring, developing during summer, maturing in boreal winter, and decaying over the following spring. This feature is referred to as ENSO phase locking. Recent studies have noted that seasonal modulation of the ENSO growth rate is essential for this process. This study investigates the fundamental effect of a seasonally varying growth rate on ENSO phase locking using a modified seasonally dependent recharge oscillator model. There are two phase locking regimes associated with the strength of the seasonal modulation of growth rate: 1) a weak regime in which only a single peak occurs and 2) a strong regime in which two types of events occur either with a single peak or with a double peak. Notably, there is a seasonal gap in the strong regime, during which the ENSO peak cannot occur because of large-scale ocean-atmosphere coupled processes. We also retrieve a simple analytical solution of the seasonal variance of ENSO, revealing that the variance is governed by the time integral of seasonally varying growth rate. Based on this formulation, we propose a seasonal energy index (SEI) that explains the seasonal gap and provides an intuitive explanation for ENSO phase locking, potentially applicable to global climate model ENSO diagnostics.
\end{abstract}

KEYWORDS: Atmosphere-ocean interaction; Climate variability; ENSO; Stability

\section{Introduction}

El Niño-Southern Oscillation (ENSO) is the strongest interannual climate phenomenon, and has significant socioeconomic impacts (Alexander et al. 2002; Timmermann et al. 2018). Its warm (cold) phase, El Niño (La Niña), tends to begin during the boreal spring, reaches a peak during boreal winter, and decays over the following spring. Such seasonally tied evolution is referred as the annual cycle amplitude/phase locking of ENSO (e.g., An and Wang 2001; Timmermann et al. 2018). This annuallocking feature is often measured by the variance or histogram of the peak calendar month (Chen and Jin 2020).

Historically, there have been two suggested mechanisms for ENSO phase locking: 1) seasonally varying ENSO instability (Philander et al. 1984; Hirst 1986; Li 1997; Galanti and Tziperman 2000; An and Wang 2001) and 2) nonlinear interactions between the intrinsic ENSO frequency and annual cycle (Jin et al. 1994; Tziperman et al. 1994). More recently, using a modified harmonic oscillator model, An and Jin (2011) showed that a model in which the frequency is modulated by the annual cycle could not simulate ENSO phase locking, while a model in which the stability is modulated by the annual cycle could simulate this phenomenon.

Later, Stein et al. (2014) and Chen and Jin (2020) confirmed that seasonal modulation of ENSO instability is key to the phase/amplitude locking of ENSO. Stein et al. (2014) tested the two leading hypotheses for the phase locking mechanism

¿ Denotes content that is immediately available upon publication as open access.

Corresponding author: Soon-Il An, sian@yonsei.ac.kr using a modified recharge oscillator model, concluding that seasonally varying growth rate is key to the seasonal variance in ENSO. Chen and Jin (2020) examined the impact of annual cycle modulation of physical processes on phase locking. They introduced a seasonal modulation of the ENSO growth rate, linear frequency, nonlinearity, and stochastic forcing, comparing these impacts on ENSO phase locking. Their conclusions are consistent with those of Stein et al. (2014), identifying that seasonal modulation of the ENSO growth rate is essential for ENSO phase locking.

Typically, the strongest ENSO instability occurs from summer to late fall (Tziperman et al. 1997; Stein et al. 2010; Jin et al. 2019). This seasonality in ENSO stability (Li 1997; Tziperman et al. 1997) is related to the seasonal migration of the intertropical convergence zone (ITCZ) (Philander 1983), and seasonal changes in thermocline depth, ocean upwelling velocity, and negative cloud feedback processes (Dommenget and $\mathrm{Yu}$ 2016; Li and Hsu 2017). The combination of these processes collectively modulates the coupling strength between the ocean and atmosphere, yielding seasonal modulation of the growth rate of ENSO [see Eq. (7) in Stein et al. (2010)]. In the context of ENSO stability, the boreal wintertime is a neutral period. As such, it is very natural to hypothesize that ENSO must reach its peak during the wintertime following the strongest seasons for instability, and that seasonal changes in ENSO stability play a major role in ENSO phase locking. Theoretical support for this hypothesis, however, has not yet been fully addressed.

This study considers the fundamental role of the seasonal modulation of the ENSO growth rate to phase locking. A conceptual model for ENSO, the "recharge oscillator model" (ROM; Jin 1997), is modified to include seasonally dependent ENSO growth rate and nonlinear physical processes. The model collectively incorporates two aspects: 1) the seasonality 
of linear feedback processes associated with the background state of the tropical Pacific and 2) nonlinear feedback processes such as nonlinear dynamical heating and westerly wind bursting (Levine and Jin 2010; Kim and An 2020). This seasonally modified ROM is used to investigate the effects of seasonally varying growth rate on seasonal variance and peak calendar month, with a combined analytical and numerical approach. We find that the time integral of the seasonal growth rate anomaly is key for phase locking.

This paper is structured as follows. Section 2 describes the seasonally modified ROM, the parameter fitting method, and the data used in this research. Section 3 demonstrates the effect of a seasonally varying growth rate on the probabilistic evolution of ENSO, focusing on seasonal variance and peak calendar month. In section 4, we retrieve an analytical formulation for seasonal variance and used this to conceptualize the influence of a seasonally varying growth rate. Section 5 investigates the effect of seasonally varying growth rate on peak calendar month, discussing the associated phase locking regimes. Section 6 shows the impact of nonlinear processes on phase locking, such as quadratic nonlinearity and multiplicative noise. Section 7 summarizes the study with a discussion.

\section{Model and data}

\section{a. Seasonal recharge oscillator model}

We adopt a recharge oscillator model (Jin 1997; Burgers et al. 2005) to investigate the effect of sea surface temperature (SST) instability on ENSO phase locking (e.g., An and Jin 2011). Based on the modified version of ROM, including quadratic and stochastic nonlinearity (e.g., Levine and Jin 2010; Kim and An 2020; An et al. 2020), the Bjerknes stability index $\left(-\lambda_{0}\right)$ is modulated by a periodic function $f(t)$ :

$$
\begin{aligned}
& \frac{d T}{d t}=-\lambda_{0}[1+f(t)] T+F h+\beta T^{2}+\sigma_{1}(1+B T) \xi_{1}, \\
& \frac{d h}{d t}=-\alpha T-\epsilon h+\sigma_{2} \xi_{2},
\end{aligned}
$$

where $T$ and $h$ are the area-averaged equatorial eastern SST anomaly (SSTA) and zonal-mean thermocline depth anomaly over the equatorial Pacific zone, respectively. We denote the seasonally modified ROM as an SROM. Now, the SROM exhibits a seasonally varying growth rate, $f(t)$, modified from the original growth rate, $-\left(\lambda_{0}+\epsilon\right) / 2$. Here, $F, \alpha$, and $\epsilon$ are parameters for the linear process, representing the efficiency of the thermocline feedback, forcing factor for oceanic wave motions, and slow oceanic adjustment process, respectively (Timmermann et al. 2018). The parameters $\beta$ and $B$ are associated with the nonlinear processes, representing quadratic nonlinearity mainly driven by nonlinear oceanic dynamical heating (Kim and An 2020) and state-dependent noise associated with SST-dependent westerly wind bursting (Levine and Jin 2010), respectively. Additionally, $\beta$ also represents the effects of nonlinear heat flux by tropical instability waves (An 2008; Xue et al. 2020) and nonlinear atmospheric convection response to SST (Takahashi and Dewitte 2016). The terms $\xi_{1}$ and $\xi_{2}$ are independent white noise with zero mean and unit variance, while $\sigma_{1}$ and $\sigma_{2}$ are their amplitudes, respectively. The noise excites and sustains the oscillation of SROM in a stable regime with a negative growth rate. The time integration of Eqs. (1a) and (1b) is carried out with a 0.01-month time step using the EulerMaruyama method. We apply the typical El Niño (La Niña) criteria for the ENSO peak detection such that three consecutive 5-month running means of SST are above (below) one standard deviation.

Then, Eqs. (1a) and (1b) are converted into the FokkerPlanck equation (FPE), which describes the time evolution of the probability density function (PDF) of $T$ and $h, p(T, h, t)$ (Risken 1989; An et al. 2020). It directly provides statistical properties of the solution, such as the mean and variance over time. As such, it is an effective tool to measure ENSO phase locking with seasonal variance and peak calendar month. Note that the FPE's PDF solution is identical to the ensemble solution of Eqs. (1a) and (1b). The FPE is written as

$$
\begin{aligned}
\frac{\partial}{\partial t} p= & -\frac{\partial}{\partial T}\left(\left\{-\lambda_{0}[1+f(t)] T+F h+\beta T^{2}+\frac{1}{2} \sigma_{1}^{2} B(1+B T)\right\} p\right) \\
& -\frac{\partial}{\partial h}[(-\alpha T-\epsilon h) p]+\frac{1}{2} \sigma_{1}^{2} \frac{\partial^{2}}{\partial T^{2}}\left[(1+B T)^{2} p\right]+\frac{1}{2} \sigma_{2}^{2} \frac{\partial^{2} p}{\partial h^{2}} .
\end{aligned}
$$

We adopt Stratonovich calculus for noise interpretation (Risken 1989). Equation (2) is solved with a central difference method using a $\Delta T=0.05 \mathrm{~K}$ and $\Delta h=0.8 \mathrm{~m}$ grid, under a rectangularshaped domain with boundaries $T=[-5,5] \mathrm{K}$ and $h=[-20,20]$ $\mathrm{m}$. Time integration is carried out using the Euler method where $d t=0.01$ month. A marginal distribution of $p[p(T)=$ $\left.\int p(T, h) d h\right]$ is calculated to obtain the variance and peak timing of ENSO. The initial conditions of $p$ are set as a two-dimensional (2D) Dirac delta function, in which their centers are located at $[T, h]=[0 \mathrm{~K}, 10 \mathrm{~m}]$ and $[T, h]=[0 \mathrm{~K},-10 \mathrm{~m}]$ for the onset of El Niño and La Niña, respectively.

\section{b. Parameter fitting}

The parameters for the SROM are obtained from the observation fitting. The linear parameters $\left(\lambda_{0}, F, \alpha\right.$, and $\left.\epsilon\right)$, noise terms $\left(\sigma_{1}\right.$ and $\left.\sigma_{2}\right)$, and seasonal SST instability $[f(t)]$ are obtained with the least squares fitting from monthly $T$ and $h$ data, following previous studies (e.g., Burgers et al. 2005; Stein et al. 2010; Kim and An 2020; An et al. 2020). The $T$ and $h$ values are averaged for the Niño- 3.4 region $\left(5^{\circ} \mathrm{S}-5^{\circ} \mathrm{N}, 170^{\circ}-120^{\circ} \mathrm{W}\right)$ and equatorial line $\left(0^{\circ}, 120^{\circ} \mathrm{E}-80^{\circ} \mathrm{W}\right)$, respectively. The details of this data are provided in section $2 \mathrm{c}$.

The linear parameters $\left(\lambda_{0}, F, \alpha\right.$, and $\left.\epsilon\right)$ are obtained by multiple linear regression between time derivative terms $\left(T_{i+1}-T_{i}\right.$ and $\left.h_{i+1}-h_{i}\right)$ with $T_{i}$ and $h_{i}$, where $i$ indicates the monthly time $(i=1,2, \ldots, n-1)$ and $n$ is the number of the data. The noise terms, $\sigma_{1}$ and $\sigma_{2}$, are calculated as the standard deviation of the residual of fitting. The nonlinear parameters, $\beta$ and $B$, are adopted from An et al. (2020), where the parameter fitting is carried out using same data, but with the Niño-3 region $\left(5^{\circ} \mathrm{S}-5^{\circ} \mathrm{N}\right.$, $150^{\circ}-90^{\circ} \mathrm{W}$ ) instead of the Niño-3.4 region. As the nonlinear feedback process is much stronger in the Niño-3 than the Niño-3.4 region (An and Jin 2004; Hayashi and Jin 2017), we utilize the nonlinear parameters obtained from the former to examine the impact of nonlinear processes. The utilized SROM parameters are 
TABLE 1. Utilized SROM parameters. Linear and noise parameters are fitted from the observation, and nonlinear parameters are adopted from An et al. (2020). Values in parentheses denote the $95 \%$ confidence interval (CI).

\begin{tabular}{|c|c|c|c|c|c|c|c|c|}
\hline \multirow[b]{2}{*}{ Parameter } & \multicolumn{4}{|c|}{ Linear component } & \multicolumn{2}{|c|}{$\begin{array}{l}\text { Nonlinear } \\
\text { component }\end{array}$} & \multicolumn{2}{|c|}{ Noise component } \\
\hline & $\begin{array}{c}\lambda_{0} \\
\left(\text { month }^{-1}\right)\end{array}$ & $\begin{array}{l}F\left(\mathrm{~m} \mathrm{~K}^{-1}\right. \\
\left.\text { month }^{-1}\right)\end{array}$ & $\begin{array}{l}\alpha\left(\mathrm{m} \mathrm{K}^{-1}\right. \\
\left.\text { month }^{-1}\right)\end{array}$ & $\begin{array}{c}\epsilon \\
\left(\text { month }^{-1}\right)\end{array}$ & $\begin{array}{l}\beta\left(\mathrm{m} \mathrm{K}^{-1}\right. \\
\left.\text { month }^{-1}\right)\end{array}$ & $\begin{array}{c}B \\
\left(\mathrm{~K}^{-1}\right)\end{array}$ & $\begin{array}{c}\sigma_{1} \\
\left(\mathrm{~K} \mathrm{month}^{-1}\right)\end{array}$ & $\begin{array}{c}\sigma_{2} \\
\left(\mathrm{~m} \mathrm{month}{ }^{-1}\right)\end{array}$ \\
\hline $\begin{array}{c}\text { Value } \\
(95 \% \text { CI })\end{array}$ & $\begin{array}{c}0.0748 \\
( \pm 0.0200)\end{array}$ & $\begin{array}{c}0.0202 \\
( \pm 0.0028)\end{array}$ & $\begin{array}{c}0.9138 \\
( \pm 0.1652)\end{array}$ & $\begin{array}{c}0.0316 \\
( \pm 0.0236)\end{array}$ & 0.012 & 0.193 & 0.23 & 1.68 \\
\hline
\end{tabular}

listed in Table 1 . In sections 3,4 , and 5 , we utilize the linear parameter settings, and exclude nonlinear parameters $(\beta=B=0)$ from the default parameter set. In section 6 , we utilize the full default parameter set, including the nonlinear parameters.

The seasonal SST growth rate $f(t)$ is estimated by multiple linear fitting from annual time series of $T$ for each calendar month of the year following Stein et al. (2010). We carry out linear regression between $T_{j+1}-T_{j}$ and $T_{j}$, where $j$ represents the month of the year $(j=1,2, \ldots, 12)$. The estimated $f(t)$ is fitted as sinusoidal function, $A \sin \left(w_{a} t+\phi\right)$ where $A=1.8, \phi=0.08 \pi$, and $w_{a}=2 \pi / 12$ month $^{-1}$ using the least squares method.

\section{c. Data}

The monthly SST anomaly $(T)$ is obtained from the National Oceanic and Atmospheric Administration (NOAA) Extended Reconstructed Sea Surface Temperature version 5 (ERSSTv5) (Huang et al. 2017). The monthly thermocline depth anomaly $(h)$ is calculated from a merged dataset of the Simple Ocean Data Assimilation version 2.2.4 (SODAv2.2.4) for 1958-2010 (Giese and Ray 2011) and the NCEP Global Ocean Data Assimilation System (GODAS) reanalysis dataset for 2011-16 (Behringer et al. 1998) using the $17^{\circ} \mathrm{C}$ isotherm criteria. The terms $T$ and $h$ are averaged for the Niño-3.4 region $\left(5^{\circ} \mathrm{S}-5^{\circ} \mathrm{N}, 170^{\circ}-\right.$ $\left.120^{\circ} \mathrm{W}\right)$ and equatorial line $\left(0^{\circ} \mathrm{N}, 120^{\circ} \mathrm{E}-80^{\circ} \mathrm{W}\right)$, respectively.

\section{Probabilistic evolution of El Niño and La Niña}

To demonstrate the effect of seasonal modulation on ENSO growth rate, the time evolution of the SSTA PDF is solved using the FPE of the SROM [Eq. (2)]. A seasonal SST instability function is set as $f(t)=A \sin \left(w_{a} t\right)$, where $A=2.0$ and $w_{a}=$ $2 \pi / 12$ month $^{-1}$ as an approximated value of the observation (see section $2 b$ ). The growth rate $-\lambda_{0}[1+f(t)]$ is the maximum (minimum) in September (March) and positive between July and November, yet the annual-mean growth rate is negative (i.e., $-\lambda_{0}<0$; e.g., Burgers et al. 2005). Therefore, with this growth rate setting, SROM produces a damped oscillation. The nonlinearities are turned off by excluding the nonlinear parameters $(\beta$ and $B)$ for simplicity. We carry out two experiments with varying SST instability $(A)$ seasonal cycle strengths, one with the seasonal cycle off $\left(A=0\right.$ and $\beta=B=0$; $\mathrm{OFF}_{-}$ LIN) and one with the seasonal cycle on $(A=2.0$ and $\beta=B=0$; ON_LIN). Note that annual mean growth rate $\left(-\lambda_{0}\right)$ is same for both on and off experiments. The integration is performed for El Niño and La Niña onset initial conditions.

The evolutions of El Niño and La Niña under stochastic noise are visualized as a time evolution of the SST anomaly
(SSTA) PDF. For OFF_LIN, El Niño and La Niña exhibit symmetric evolution over time (Fig. 1a). The mean SSTA (black line) begins to increase upon initiation, and peaks eight months after its onset. Following the peak, a decaying process begins, and the ENSO phase turns to the opposite phase. As cyclic oscillation occurs, the SSTA amplitude decreases gradually, eventually converging into a steady state with a zero mean. This mean solution is identical to the one in the SROM with no noise $\left(\sigma_{1}=\sigma_{2}=0\right)$, that is, a damped oscillation resulting from large-scale, low-frequency coupled ocean-atmosphere processes. Stochastic noise produces an ensemble spread of SSTA around the mean solution. The instantaneous standard deviation of SSTA steadily increases from initiation, reaching a steady state approximately 1.5 years after initiation. An analytical solution of the standard deviation for the steady state (Kim and An 2020) is as follows:

$$
\sigma_{\text {mean }}^{2}=\sqrt{\frac{\left(\alpha F+\epsilon \lambda_{0}+\epsilon^{2}\right) \sigma_{1}^{2}+F^{2} \sigma_{2}^{2}}{2\left(\lambda_{0}+\epsilon\right)\left(\alpha F+\epsilon \lambda_{0}\right)}} .
$$

When the seasonal cycle of SST instability is included, the overall evolution of ENSO is modulated from the early development phase to a steady state, but the symmetric evolution of El Niño and La Niña is maintained (Fig. 1b). Comparing ON_LIN and OFF_LIN, the developmental pattern becomes less straightforward, and the peak timing shifted from 8 to 11 months. Notably, the standard deviation does not converge into a stationary state; rather, it becomes a cyclostationary state with a 12-month period. The frequency of the standard deviation is synchronized with a seasonally varying growth rate, producing the phase locking of ENSO. Even if the growth rate turns positive for several months (July to November), the overall oscillation is within a stable regime, rather than being unstable with infinite growing SSTA (Fig. 2). In section 4, we will clearly demonstrate that the annual mean growth rate is key to determining the overall stability of ENSO, as opposed to the seasonal anomaly of the growth rate. Finally, the effect of the seasonal cycle of the growth rate can be characterized by two features: 1) the modulation of the standard deviation of SSTA on each calendar month and 2) the modulation of ENSO development and peak manifested by the mean SSTA solution.

\section{Seasonal modulation of ENSO variance}

We propose an analytical solution for SROM to understand the fundamental role of the seasonally varying growth rate on ENSO variance. For seasonally varying SST instability, 
(a)

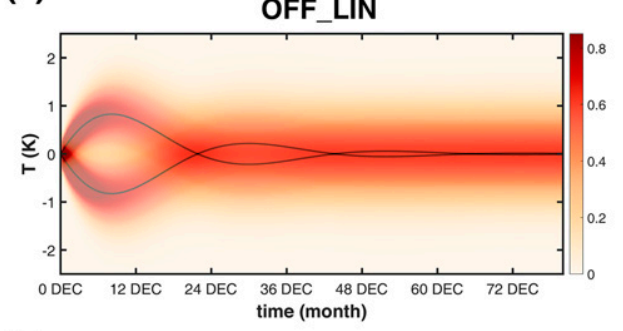

(c)

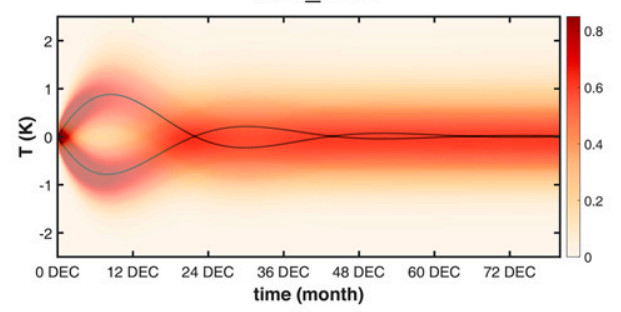

(e)

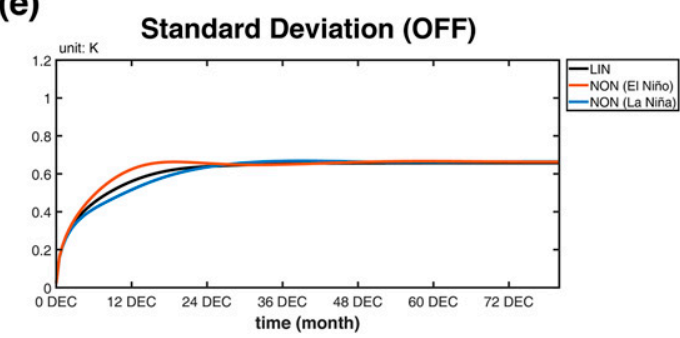

(b)

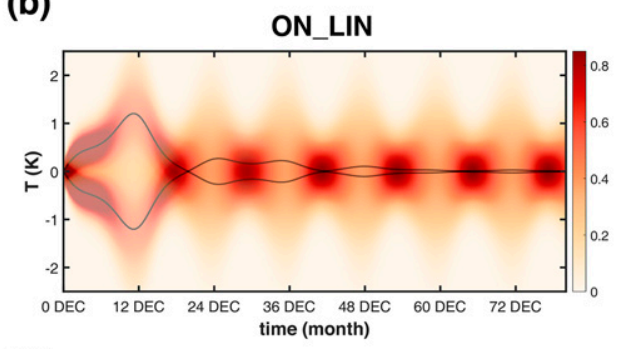

(d)

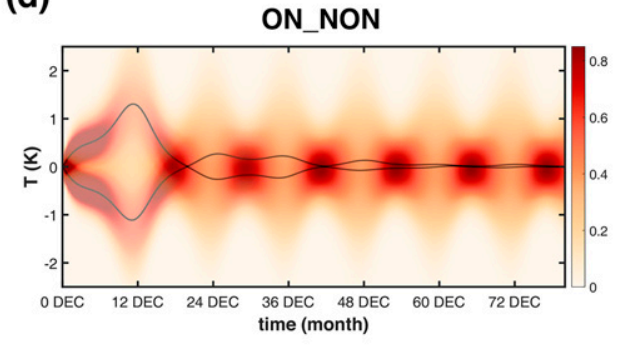

(f)

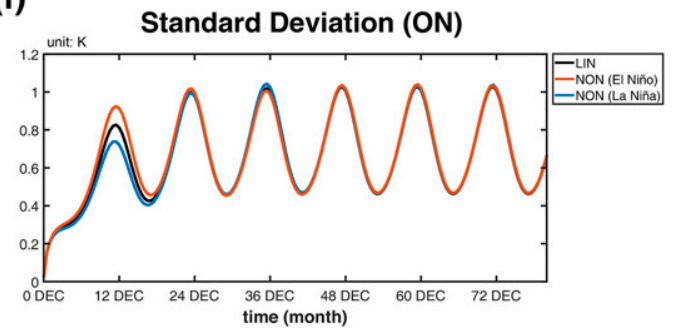

FIG. 1. (a) Evolution of the SSTA PDF obtained from the OFF_LIN experiment over time. The red shading and thick black line denotes the marginal distribution $\left[p(T)=\int p(T, h) d h\right]$ and mean $\left[\int T p(T) d T\right]$ for SSTA, respectively. The solutions for El Niño and La Niña overlap. (b)-(d) As in (a), but for ON_LIN, OFF_NON, and ON_NON, respectively. (e) The time evolution of the standard deviation of SSTA (unit: K) for the OFF experiments $(A=0)$. Note that the solution for the linear El Niño and La Niña experiments overlap on the black line as they are identical. (f) As in (e), but for the ON experiments $(A=2)$.

$-\lambda_{0}[1+f(t)]$, where $f(t)$ is an arbitrary periodic function, a cyclostationary solution of the linear SROM [Eqs. (1a) and (1b) with $\beta=B=0]$ ) is approximately solved with a perturbation method (see appendix A).

The approximate seasonal variance solution reads

$$
\sigma_{T}^{2}(t) \approx \sigma_{\text {mean }}^{2}\left[1-2 \lambda_{0} \int f(t) d t\right],
$$

where $\sigma_{\text {mean }}^{2}$ is obtained from Eq. (3). Thus, seasonal variance equals to the modulation of annual mean variance, $\sigma_{\text {mean }}^{2}$ by the time integral of the seasonal growth rate anomaly, $\int f(t) d t$. Assuming a growth rate anomaly, $f(t)=A \sin \left(w_{a} t\right)$, where $w_{a}=$ $2 \pi / 12$ month $^{-1}$, with a maximum in September, the solution then becomes

$$
\sigma_{T}^{2}(t) \approx \sigma_{\text {mean }}^{2}\left[1+\frac{2 \lambda_{0} A}{w_{a}} \cos \left(w_{a} t\right)\right] .
$$

Again, seasonal variance sinusoidally oscillates with its amplitude, $\sigma_{\text {mean }}^{2}\left(2 \lambda_{0} A / w_{a}\right)$ with a 12 -month period. Integrating the sinusoidally varying SST instability yields an exact +3 months between the growth rate and variance; thus, the maximum variance occurs in December. The obtained analytical solution clearly explains the occurrence of the maximum variance in the boreal winter, after a maximum growth rate in the summer to fall (see Fig. 1j of Timmermann et al. 2018). Compared with the numerical solution obtained from FPE [Eq. (2)], the analytical solution somewhat underestimates the variance over the season and has a phase delay (Fig. A1). Increasing $A$ results in a rise in the underestimation $\left(\sim 0.02,0.05\right.$, and $0.1 \mathrm{~K}^{2}$ for $A=0.5$, 1.0 , and 2.0 , respectively), while there are only relatively small changes to the phase delay ( $\sim 0.5$ months). However, its overall range is in good agreement, making the solution reasonable. The strength of seasonal locking is measured by the standard deviation of $\sigma_{T}^{2}(t)\left[\left(\sigma_{\text {mean }}^{2} / \sqrt{2}\right)\left(\lambda_{0} A / w_{a}\right)\right]$, which increases with the annual mean SST instability $\left(\lambda_{0}\right)$, seasonal amplitude of growth rate $(A)$, and annual mean variance $\left(\sigma_{\text {mean }}^{2}\right)$.

The observed seasonal SST growth rate exhibits sinusoidal oscillation over the seasons with a maximum in August and a minimum in February (Fig. 2) (see section $2 b$ for the estimation method). Seasonal variance is reproduced using the analytical solution [Eq. (4)] with fitted parameters, as shown in Fig. 2 b. 
(a)

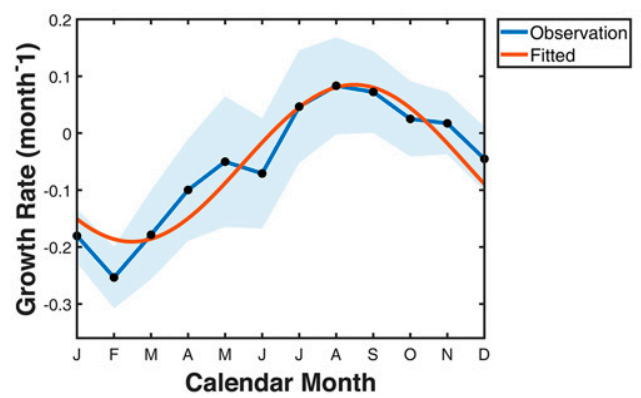

(b)

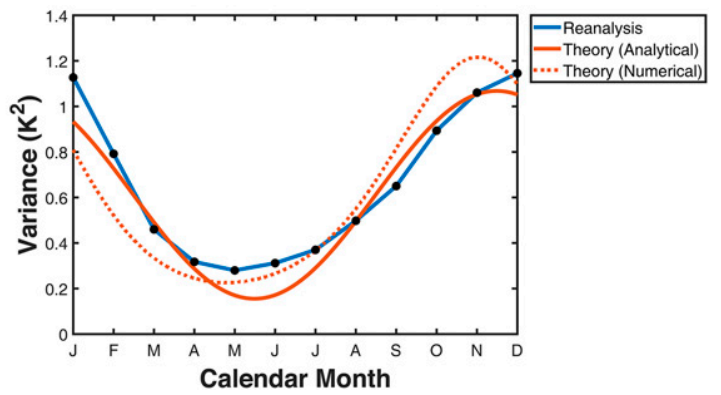

FIG. 2. (a) Estimated seasonal SST instability (growth rate) for the observations (blue line), and fitted sine curve (orange line) (unit: month $^{-1}$ ). The blue shade denotes the $95 \%$ confidence interval for the estimated seasonal SST growth rate. (b) The observed seasonal variance of the Niño-3.4 index (blue line) and the reproduced variance via the analytical solution (solid orange line) and numerical solution (dashed orange line) with the fitted growth rate in (a) (unit: $\left.\mathrm{K}^{2}\right)$. The numerical solution is obtained from the FPE [Eq. (2)] with the linear version of parameters $(\beta=$ $B=0)$.

The observational features, such as the maximum/minimum timing and intensity, are well reproduced by the analytical solution. Specifically, the reproduced variance shows a maximum (minimum) between November and December (May and June), while the observations had a maximum (minimum) in December (May). A variance underestimation by approximately $0.5 \mathrm{~K}^{2}$ in the boreal spring and summer (March-August) is also observed for the reproduced solution.

For ROM, the $T$ and $h$ variables can be viewed as the momentum $(P)$ and position $(x)$ of a classical damped oscillator, where $T \rightarrow P$ and $h \rightarrow x$ (Burgers et al. 2005). The energy of such system is written as, $E=P^{2} / 2 m$, where $m$ is its mass. Thus, the SST variance $\left(\left\langle T^{2}\right\rangle\right)$ can be regarded as the ensemble mean energy of the system. As such, the role of $-\lambda_{0} \int f(t) d t$ in Eq. (4) can be interpreted as the seasonal energy modulation driven by the seasonally varying growth rate. Here, we define the integral term as the seasonal energy index (SEI):

$$
\text { Seasonal Energy Index }=-\lambda_{0} \int f(t) d t \text {. }
$$

The SEI varies seasonally, and a high (low) SEI state indicates high (low) seasonal energy. A detailed explanation and application of the SEI is provided in section 5.

\section{Modulation of the ENSO peak calendar month}

In this section, we investigate the role of the seasonally varying growth rate on the ENSO peak calendar month. The FPE [Eq. (2)] is solved for $f(t)=A \sin \left(w_{a} t+\varphi\right)$, excluding nonlinear parameters $(\beta=B=0)$. Two experiments are carried out by varying the seasonal cycle strength of the SST instability: 1$)$ seasonal cycle off $(A=0)$ and 2$)$ seasonal cycle on $(A=2)$. We calculate the peak calendar month alongside the onset calendar month. The calendar month is labeled on the time frame $(t)$, based on $f(t)=A \sin \left(w_{a} t\right)$, where the maximum (minimum) growth rate occurred in September (March). The onset calendar month is varied with the phase of seasonal growth rate $(\varphi)$, ranging from 0 (January) to $2 \pi$ (January) with a $2 \pi / 1920$ month $^{-1}$ interval (see upper panels in Fig. 3 for an example). We exclude the noise $\left(\sigma_{1}\right.$ and $\left.\sigma_{2}\right)$, to focus on the interaction between large-scale ocean-atmosphere coupled processes with seasonal varying background state. This is carried out by considering the mean of the FPE solution, identical to the one in the SROM without noise [Eqs. (1a) and (1b) with $\sigma_{1}=\sigma_{2}=0$ ]. A peak is defined as the local maximum point of the mean SSTA. The impact of noise will be discussed at the end of the section in detail.

As a first step, we demonstrate the evolution of the mean SSTA $\left[\int T p(T, t) d T\right]$ for two different onset calendar months, March $(\varphi=0)$ and September $(\varphi=\pi)$. Figure 3 presents the growth rate and the following mean evolution of the SSTA. For the March onset experiment, the mean SSTA peaks in early November with $1.4 \mathrm{~K}$ for the seasonal cycle on $(A=2)$, whereas it is $0.8 \mathrm{~K}$ in late October for the seasonal cycle off $(A=0)$. Seasonal modulation of the growth rate results in a slight delay in a peak timing (calendar month) and an intensification of the amplitude. For the September onset experiment, the mean SSTA features double peak in January with $0.7 \mathrm{~K}$ and November with $0.9 \mathrm{~K}$ for seasonal cycle on $(A=2)$, whereas it peaks in April at $0.8 \mathrm{~K}$ for seasonal cycle off $(A=0)$. The double peak feature is a result of the seasonal modulation of the growth rate. The double peak of ENSO can be found in observation as well (Cole et al. 2002; Okumura and Deser 2010; Park et al. 2021). We classify these two peaks based on their intensities as the primary and secondary peaks. For the September onset experiment, the peaks in January and November are classified as the primary and secondary peaks, respectively.

Figure 4 illustrates the peak calendar month and intensity along with the onset calendar month spanning all months (January to January). For the no seasonal cycle case $(A=0)$, the El Niño and La Niña peaks occur approximately 8 months after initiation, regardless of the onset month (Fig. 4a). All seasons share equal peak intensity during the onset calendar month (Fig. 4b); however, with the inclusion of the seasonal cycle $(A=2)$, the peak month exhibits a nonlinear relationship 
(a)
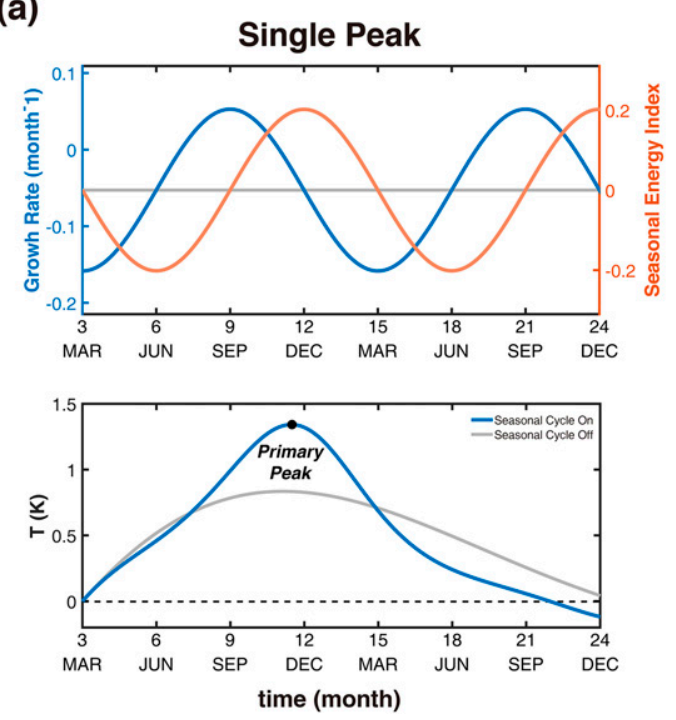

(b)
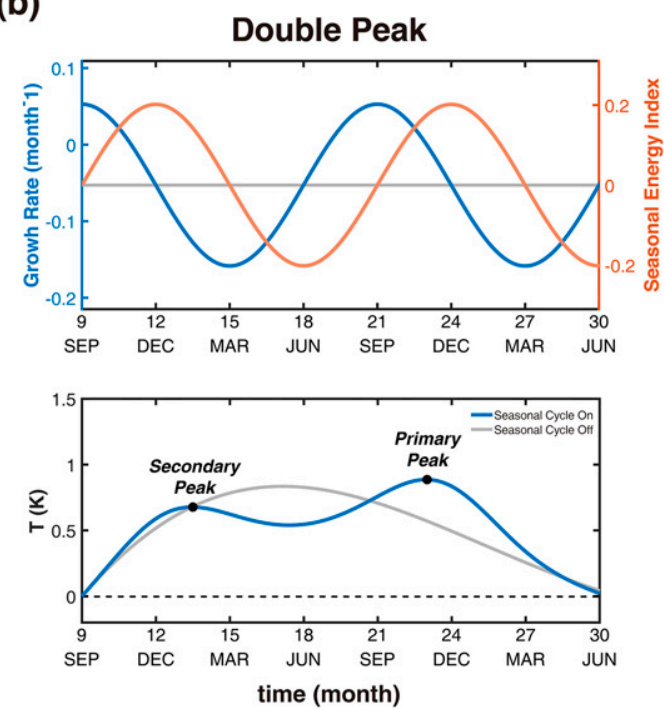

FIG. 3. Example of the experiment settings and results. (a) (top) The growth rate $\left\{-\lambda_{0}[1+f(t)]\right\}$ and SEI $\left[-\lambda_{0} \int f(t) d t\right]$ are presented as the blue and orange lines, respectively. The onset calendar month is set to March. (bottom) The time series of mean SSTA solved by the FPE [Eq. (2)] with given seasonal growth rate is denoted by the blue line. The mean SSTA exhibits a single peak during the first cycle, and is marked as the primary peak. For both panels in (a), the result for the no seasonal cycle $[f(t)=0]$ is presented as the gray line. (b) As in (a), but the onset calendar month is September. The mean SSTA features a double peak, marked as the primary and secondary peaks.

with its onset month. For an April onset, a typical calendar month for ENSO initiation, the peak occurs in December, consistent with the observations. The largest peak intensities are observed in a narrow range during November and December. A seasonally varying growth rate provides favorable conditions for ENSO development in the boreal winter (NovemberJanuary), in terms of intensity and peak timing.

Notable novel features are found for seasonality in terms of peak timing. First, a double peak of El Niño and La Niña appears with the inclusion of the seasonal cycle, while only a single peak appears for cases excluding the seasonal cycle. The secondary peak appears only if the event is initiated between June and October; otherwise, the event only features a single peak. The secondary peak occurred in January-April and AugustNovember, while the primary peak occurs in November-January. By definition, the secondary peak intensity is always weaker than that of the primary peak. Second, there are months during which neither a primary nor secondary peak occurs. From April to August, a jump of the peak month occurs, producing a gap for the period. Here, we denote this gap as the seasonal gap of ENSO. The seasonal gap represents an impossible (or very unlikely) regime for ENSO peaks driven by large-scale, ocean-atmosphere coupled processes in the absence of atmospheric noise.

Next, we perform an identical experiment with different $A$ values ranging from 0 to 2.5 , with a 0.01 interval. Figure 5 illustrates the set of possible peak calendar months along $A$ with its intensity as an extension of Fig. 4b. Two distinct regimes appear along $A$, characterized by a seasonal gap and a doublepeak feature. For $A<0.5$ (weak regime), all ENSO events have a single peak that can occur at any month in the year, while for $A>0.5$ (strong regime), the seasonal gap appears as well as double-peak events. As $A$ increases, the seasonal gap symmetrically expands from around June, while the primary peak regime shrinks alongside it. The impossible regime zone becomes wider and the possible peak month range is narrowed to the boreal winter (DJF) as $A$ increases. The observed tropical Pacific is in strong regime $(A=1.8)$ where primary peak occurs in boreal winter and has seasonal gap in April to August.

The emergence of a seasonal gap is a result of the bifurcation of the peak time induced by $A$, and it can be obtained by solving Eq. (B6) in appendix B. This equation, however, is extremely complicated to solve and so it is not necessary to provide the details about these types of gaps. Alternatively, such behavior can be qualitatively understood in terms of the SEI, introduced in section 4. Using Eq. (6), the SEI of the system is written as

$$
\mathrm{SEI}=\frac{2 \lambda_{0} A}{w_{a}} \cos \left(w_{a} t\right)
$$

The SEI oscillates over the calendar months with a minimum (maximum) in June (December) with $-2 \lambda_{0} A / w_{a}\left(2 \lambda_{0} A / w_{a}\right)$ (see Fig. 3). We assume that there is a hypothetical SEI threshold below which the ENSO peak cannot appear, leading to a seasonal gap. When the SEI of a calendar month is below (above) this threshold, the seasonal gap would (would not) appear because of its low (high) seasonal energy. As the SEI is at a minimum in June, the seasonal gap begins to appear in 
(a)

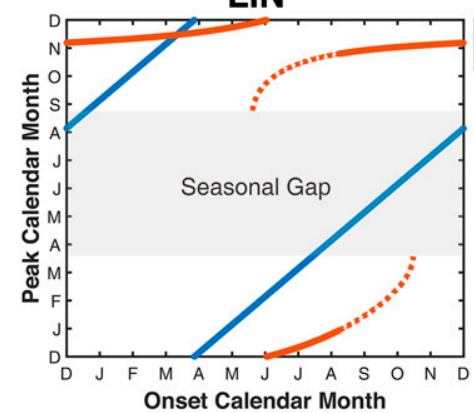

(c)

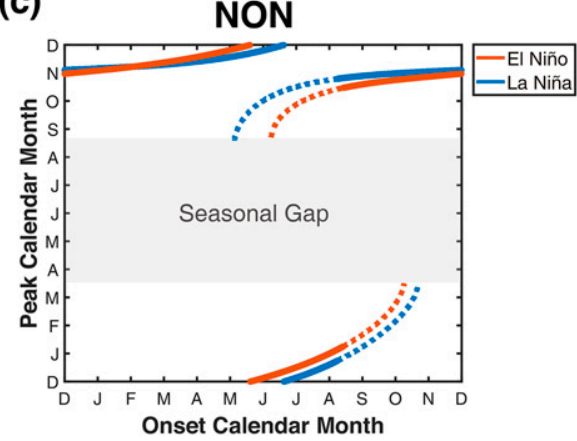

(b)

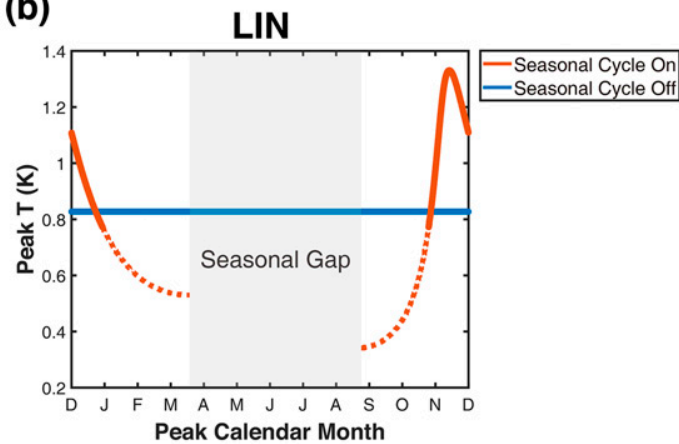

(d)

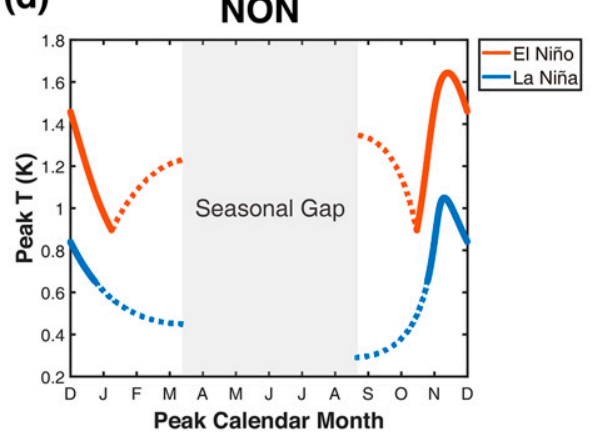

FIG. 4. (a) Peak calendar month plotted alongside the onset month for the linear experiment. The thick and dashed lines denote the primary peak (maximum) and secondary peak solutions, respectively. The orange and blue lines denote the results for the seasonal cycle in the on (ON_LIN) and off (OFF_LIN) experiments, respectively. The seasonal gap period is shaded as gray. Note that the results for El Niño and La Niña overlap on the same line as they are identical. (b) As in (a), but for the SST value at the peak calendar month. (c) As in (a), but for the nonlinear experiment with the added case of a seasonal cycle. The orange and blue lines denote the results for El Niño and La Niña onset conditions, respectively. (d) As in (c), but for the peak SST value along with the peak calendar month.

June as $A$ increases. The SEI framework is schematically illustrated in Fig. 6. A critical condition of $A\left(A^{*}\right)$ for the seasonal gap bifurcation is approximately retrieved as follows (see appendix B):

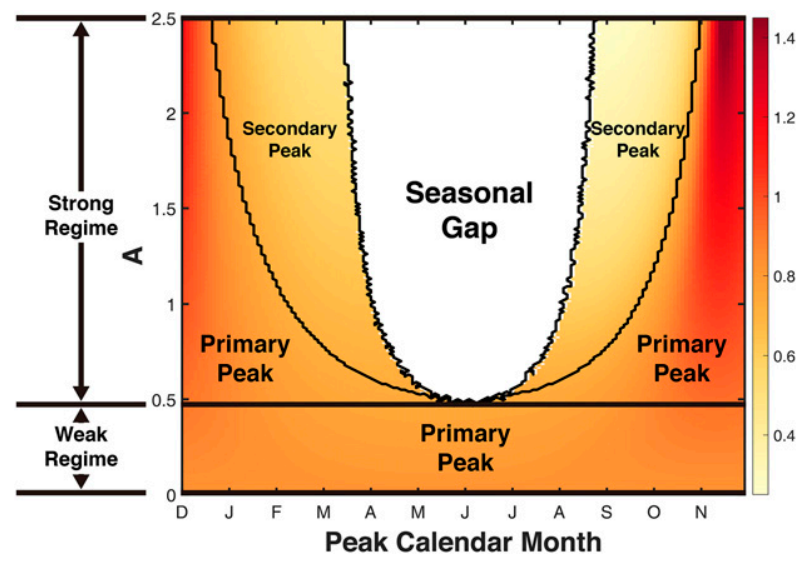

FIG. 5. A regime diagram for the peak calendar month as a function of $A$. We conduct the sensitivity experiment with varying $A$ as an extension of the results in Fig. 4b. The distinct regimes for the peak calendar month are denoted as the primary peak (maximum), secondary peak, and seasonal gap. The color denotes peak intensity (unit: K).

$$
A^{*} \approx \frac{w_{o}^{2}}{\lambda_{0} \sqrt{\frac{9}{16} w_{a}^{2}+\frac{1}{4}\left(\lambda_{0}-\epsilon\right)^{2}}},
$$

where $w_{o}$ is the ENSO intrinsic linear frequency $\left[w_{o}^{2}=\alpha F-\right.$ $\left.(1 / 4)\left(\lambda_{0}-\epsilon\right)^{2}\right]$. Note that $A^{*}$ depends on the ENSO intrinsic frequency $\left(w_{o}\right)$ and annual mean growth rate $\left(\lambda_{0}\right)$, indicating that it is directly related to the background state of the equatorial Pacific. The analytical solution [Eq. (8)] and numerically obtained $A^{*}$ are compared under various $w_{o}$ conditions by

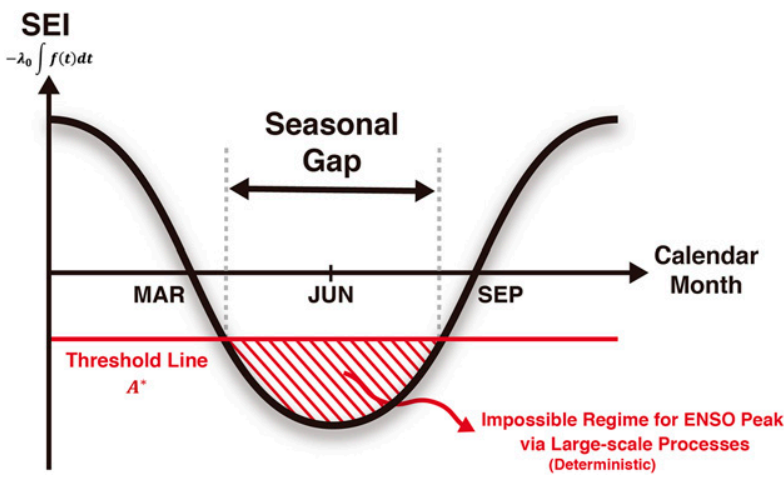

FIG. 6. Schematic concept of the seasonal gap of ENSO. 
adopting the approximated version of SROM (see appendix B for the detailed method). A quadratic dependency on $w_{o}$ is captured well by the analytical solution (Fig. B1), but there is a significant difference between numerical and analytical solution. This indicates that the adopted approximation for the analytical solution is rather crude. To utilize Eq. (8) for practical purposes, an additional correction should be carried out by multiplying a constant with Eq. (8).

The SEI can also explain the overall seasonal evolutionary features, including the double peak. Figure 3 demonstrates that primary and secondary peaks occur when the SEI is anomalously high (SEI >0), from September to March. Although there are some delays between the peak timing of SEI and mean SSTA, the period of high SEI coincides well with the El Niño peak. As $A$ increases, the peak calendar month becomes more constrained to the positive SEI state period from September to March (Fig. 5). The high anomalous seasonal energy state provides favorable conditions for the peak, by modulating the evolution path of El Niño. From this perspective, the emergence of a double peak is also attributable to the SEI. Within the first cycle of the period ( $\sim 21$ months), the SEI exhibits single or double peaks, depending on the onset calendar month (see Fig. 3). Under strong seasonal cycle condition $\left(A>A^{*}\right)$, the double-peaked SEI yields a double peak feature of SSTA.

Note that in this paper the ENSO peak is defined as the local peak of the mean PDF of $T$. As the mean PDF trajectory is identical to the no-noise solution of SROM, the peak discussed is from a deterministic, large-scale, ocean-atmosphere coupled process, as opposed to noise. Therefore, the seasonal gap does not strictly guarantee that the ENSO peak does not occur during that period. There may be a chance that the peak occurs within the seasonal gap via noise. To examine the effect of noise, we carry out an additional experiment on noise. Equations (1a) and (1b) are directly integrated over time using the same parameter set as the previous experiments. The integration is carried out for 60 years with $N=10000$ ensemble, and a histogram of peak calendar month of first El Niño is obtained along with the onset calendar month. The detailed time integration method and ENSO event criteria are outline in section $2 \mathrm{a}$. Figure 7 shows the result as an extended version of Fig. 4a with noise. The probability of the peak occurring within the seasonal gap is very low compared to the probability of occurrence during the boreal winter. Here, the noise amplitude of SSTA $\left(\sigma_{1}\right)$ $\left(\sim 0.23 \mathrm{~K} \mathrm{month}^{-1}\right)$ is based on the observations. With such a reasonable choice of noise characteristic, the seasonal gap structure is still not changed noticeably.

We also examine the sharpness of the transition between the weak and strong phase locking regimes under the influence of noise. The solid boundary between the two phase locking regimes $\left(A^{*}\right)$ would be blurred by noise. We conduct time integration of the noise SROM [Eqs. (1a) and (1b)] with varying $A$ and $w_{o}$; the details of the experimental setup are provided in the caption for Fig. B1. The result is shown in Fig. B1 overlapped with numerical/analytical $A^{*}$. The gradual transition of the regime is visualized with the strength of the seasonal gap, quantified as the minimum PDF value of the peak calendar month. A lower (higher) value signifies proximity to a solid strong (weak) phase locking regime, which features the (no) seasonal gap. The transition between the two regimes becomes

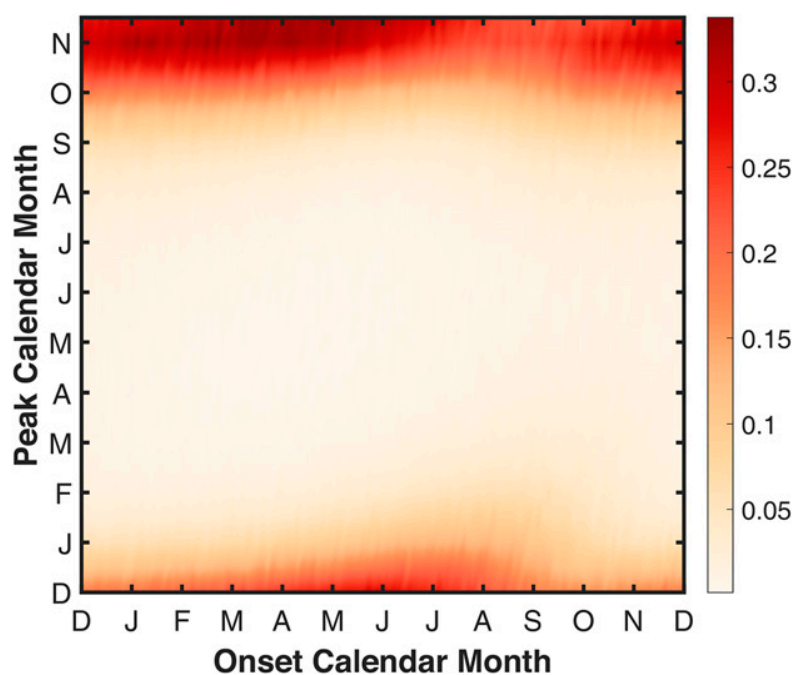

FIG. 7. The probability of an ENSO peak occurring at given calendar month, as a function of the onset calendar month. The color denotes the PDF of the peak calendar month for a given onset calendar month. Equations (1a) and (1b) are solved with an $N=$ 10000 ensemble, excluding the nonlinear parameters $(\beta=B=0)$. The peak is obtained from an El Niño (La Niña) event criteria outlined in the main text. As the solutions for El Niño and La Niña are identical in this model, we have only presented the results for the El Niño case.

gradual by noise as anticipated, and $A^{*}$ no longer serves as a strict regime boundary. For observed $w_{o}$, the seasonal gap strengths at $A^{*}$ and $2 A^{*}$ are approximately 0.03 and 0.01 , respectively. The sharpness of the transition may depend on the SROM parameters, especially for noise amplitude.

\section{Effect of nonlinear processes}

The nonlinear processes are known to be nonessential for phase locking (Chen and Jin 2020); however, it is worthwhile to discuss the effects of these processes to understand the different evolutions of El Niño and La Niña. To undertake this, we conduct a series of identical experiments, as outlined in sections 3 and 5 , the only difference being that the nonlinear parameters $(\beta$ and $B)$ are considered.

Following the experiment in section 3, we calculate the time evolution of the SSTA PDF with the inclusion of nonlinear parameters. Two experiments are carried out: 1$)$ seasonal cycle off $(A=0 ;$ OFF_NON $)$ and 2$)$ seasonal cycle on $\left(A=2.0 ; \mathrm{ON}_{-}\right.$ NON). Comparison of OFF_NON and ON_NON shows that the role of the seasonal cycle is still similar in the presence of nonlinear processes, that is, modulating the peak calendar month and facilitation of cyclostationary state of variance (Figs. 1c,d). However, the symmetric evolution of El Niño and La Niña is broken by nonlinear processes. At the peak, the mean and standard deviation are larger for El Niño than for La Niña in both nonlinear experiments (OFF_NON and ON_NON). Nonlinear dynamic heating (NDH) and westerly wind bursting (WWB) are largely responsible for these asymmetries (Levine and Jin 2010; Kim and An 2020; An et al. 
2020). In contrast, comparison of the linear (OFF_LIN and ON_LIN) and nonlinear (OFF_NON and ON_NON) experiments shows that the peak timing and variance in a (cyclo)stationary state are almost identical. This indicates that the analytical solution for seasonal variance [Eqs. (4) and (5)] derived from the linear model is still valid for the nonlinear model.

We also investigate the peak calendar month for the nonlinear model following the experiment in section 5 . We conduct experiments for the El Niño and La Niña onset conditions with inclusion of nonlinear parameters and set $A=2$. Figures $4 \mathrm{c}$ and $4 \mathrm{~d}$ present the results, denoted as NON. A slight split for the El Niño and La Niña peak months occurs when using nonlinear parameters. For an event occurrence peak in November-April, the primary and secondary El Niño peaks have been delayed compared to those of La Niña, and the reverse occurs for an AprilNovember peak event. The splitting effect is nonlinear during the onset month, although it is not significant ( $\sim 0.5$ months). In addition, the seasonal gap structure is merely changed by nonlinear parameters compared to the linear experimental result (LIN). The peak intensity of El Niño is much larger than that of La Niña for the primary and secondary peaks. For the primary peak, the El Niño is larger than La Niña by approximately $0.6 \mathrm{~K}$. The secondary peak for El Niño increases as it approaches the seasonal gap, whereas it decreases for La Niña and LIN. A detailed discussion of this phenomenon is beyond the scope of this paper.

\section{Summary and discussion}

This study explores the fundamental effects of the seasonally varying growth rate of ENSO on phase locking behavior based on variance and peak time. Through a series of numerical and analytical analyses, we establish the novel theoretical framework for ENSO phase locking. We found the seasonal gap of ENSO during which its peak cannot occur when the seasonal modulation of growth rate is strong enough. Specifically, two regimes for phase locking arise depending on the strength of the seasonal growth rate modulation: 1) a weak regime in which only a single peak occurs and 2) a strong regime characterized by a seasonal gap and a double-peak feature. Through analytical formulation of the seasonal variance, we find that the time integral of the growth rate or "seasonal energy index" (SEI) is a useful tool to characterize ENSO phase locking with peaks that tend to occur toward the largest SEI. The SEI quantifies the seasonal energy state of ENSO and provides a conceptual picture of the seasonal gap, highlighting the seasonally tied evolution of ENSO. We also confirm that nonlinear processes merely impact the peak calendar month and seasonal gap structure.

Our theory provides the simplest picture to understand the ENSO phase locking phenomenon in the context of existing literature. In previous studies (An and Jin 2011; Stein et al. 2014), an analytical solution for variance using SROM was derived, but with different physical assumptions as follows:

$$
\sigma_{T}^{2}(t) \approx \frac{1-2 \lambda_{0} B^{*} \sin \left(w_{a} t\right)+\lambda_{0}^{2} B^{* 2} \sin ^{2}\left(w_{a} t\right)}{2},
$$

where $B^{*}=\left(w_{a}^{2}-2 w_{o}^{2}\right) /\left(w_{a}^{3}-4 w_{o}^{2} w_{a}\right)$ with SST instability, $\lambda(t)=\epsilon \cos \left(w_{a} t\right)(0<\epsilon \ll 1), F=w_{o}, \alpha=w_{o}$, and $\epsilon=0$. The main difference from this study is that these studies assumed an annual mean growth rate of zero $\left(\lambda_{0}=0\right)$, such that ENSO remains in a neutral growth/damping regime, and also dismissed the noise (i.e., $\sigma_{1}=\sigma_{2}=0$ ). As the observation exhibits a negative (nonzero) annual mean growth rate and noise amplitude to some degree (nonzero), the solution presented in this study [Eq. (5)] realistically reflects the observation compared to the previous one [Eq. (9)] using a simpler form. The generalized variance solution [Eq. (4)] and the SEI framework (Fig. 6) also provide intuitive explanations of phase locking using a theoretical approach. The simplicity and generality of this study may be useful for Coupled Model Intercomparison Project (CMIP) diagnoses on ENSO simulations and phase locking projections into the future.

However, this study neglects specific processes such as colored noise (i.e., red noise) and cubic nonlinearity. The red noise represents atmospheric processes with finite memory time scale, and the cubic nonlinearity describes saturated subsurface temperature dependency on thermocline depth (Battisti and Hirst 1989; Jin 1997). These processes are known to affect ENSO properties such as annual variance and skewness (Levine and Jin 2010). Additionally, we do not consider the seasonality of another linear process $(F, \alpha$, and $\epsilon)$, noise amplitude $\left(\sigma_{1}\right.$ and $\left.\sigma_{2}\right)$, nonlinearity $(\beta)$, and multiplicative noise forcing $(B)$. These missing physical processes and seasonality may be responsible for the small differences between the theory and observation of variance (Fig. 2). Previous study (Stein et al. 2010) showed that the seasonality of $F$ and $\alpha$ could not significantly affect ENSO phase locking. In addition, the nonlinear parameters $(\beta$ and $B)$ are not dominant on ENSO variance (Fig. 1). These results indicate that these processes are not dominant players in phase locking. However, as the role of unconsidered processes is unclear, it warrants an in-depth investigation in the future.

The double peak ENSO observed in our experiment indicates an event that starts late in the year and lasts for 2 years. Its first peak occurs at a boreal winter, of which the amplitude is small because of little time to grow; and then it temporary decreases due to the weaker instability. In the following year, it starts to regrow and marks the second peak at the boreal winter. Such a long persistent double peak feature can be found in observation, especially for cold events, so-called multiyear La Niña events (Cole et al. 2002; Okumura and Deser 2010). For example, Okumura and Deser (2010) argued that the Indian Ocean capacitor effect (Xie et al. 2009) weakens ENSO-induced western Pacific winds during the mature phase of ENSO through atmospheric teleconnection, and this remote impact is more effective during La Niña, because La Niñainduced easterly winds are located in the western Pacific whereas El Niño-induced westerly winds are located in the central Pacific. Park et al. (2021) suggested that the multiyear La Niña is related to a strong connection between the tropical Pacific and midlatitude Pacific through the Pacific meridional mode, which weakens the off-equatorial wind stress curl over western Pacific as well as consequent oceanic heat recharge leading a weaker termination of the first peak event. Such complex physical processes can be incorporated to single SEI framework, indicating anomalously high SEI due to a weakened SST damping process after first peak (Fig. 3). However, 
our study cannot explain the asymmetry between El Niño and La Niña, with a much more frequent double peak of La Niña than El Niño (Figs. 4c,d). In addition, the regime diagram (Fig. 5) indicates that the phase locking and double peak share the same fundamental mechanism, even though they are apparently different phenomena. Our novel SEI framework may be useful to understand double peak event as well as phase locking, and thus to untangle the temporal complexity of ENSO within a unified picture.

Acknowledgments. This research was supported by a National Research Foundation of Korea (NRF) grant funded by the Korean government (MSIT) (NRF-2018R1A5A1024958, NRF2020R1A2B5B01094934). There are no conflicts of interest to declare.

Data availability statement. All datasets used in this research may be accessed via the following websites: ERSST, https://www1.ncdc.noaa.gov/pub/data/cmb/ersst/v5/netcdf/; SODA, https://coastwatch.pfeg.noaa.gov/erddap/griddap/ hawaii_d90f_20ee_c4cb.html; and GODAS, https://psl.noaa.gov/ data/gridded/data.godas.html.

\section{APPENDIX A}

\section{Derivation of the Seasonal Variance}

In this section, we derive the variance of the SROM. The time evolution of the statistical moment for the linear form of Eqs. (1a) and (1b) (i.e., $\beta=0$ and $B=0$ ) is obtained using Eq. (5.47) in Särkkä and Solin (2019). The second-order moment is

$$
\begin{aligned}
& \frac{d}{d t}\left\langle T^{2}\right\rangle=-2 \lambda_{0}\left\langle T^{2}\right\rangle+2 F\langle T h\rangle+\sigma_{1}^{2}-2 \lambda_{0} f(t)\left\langle T^{2}\right\rangle, \\
& \frac{d}{d t}\langle T h\rangle=-\alpha\left\langle T^{2}\right\rangle-\left(\lambda_{0}+\epsilon\right)\langle T h\rangle+F\left\langle h^{2}\right\rangle-\lambda_{0} f(t)\langle T h\rangle
\end{aligned}
$$

$$
\frac{d}{d t}\left\langle h^{2}\right\rangle=-2 \alpha\langle T h\rangle-2 \epsilon\left\langle h^{2}\right\rangle+\sigma_{2}^{2}
$$

where the angle brackets denote an ensemble mean. Denoting $x=\left\langle T^{2}\right\rangle, y=\langle T h\rangle$, and $z=\left\langle h^{2}\right\rangle$,

$$
\begin{aligned}
& \frac{d x}{d t}=-2 \lambda_{0} x+2 F y+\sigma_{1}^{2}-2 \lambda_{0} f(t) x, \\
& \frac{d y}{d t}=-\alpha x-\left(\lambda_{0}+\epsilon\right) y+F z-\lambda_{0} f(t) y, \\
& \frac{d z}{d t}=-2 \alpha y-2 \epsilon z+\sigma_{2}^{2} .
\end{aligned}
$$

A perturbation method is adopted to solve the nonhomogeneous linear time-varying parameter, ordinary differential equation (ODE). Let $\lambda_{0} f(t)=\epsilon g(t)$; then, the solution $\mathbf{x}=(x, y, z)$ is expanded as $\mathbf{x}=\mathbf{x}_{0}+\epsilon \mathbf{x}_{1}+\epsilon^{2} \mathbf{x}_{2}+\cdots$. Our goal is to obtain a solution for $x$ in a cyclostationary state $(t \rightarrow \infty)$.

The equations for the zeroth order are

$$
\begin{aligned}
& \frac{d x_{0}}{d t}=-2 \lambda_{0} x_{0}+2 F y_{0}+\sigma_{1}^{2}, \\
& \frac{d y_{0}}{d t}=-\alpha x_{0}-\left(\lambda_{0}+\epsilon\right) y_{0}+F z_{0}, \\
& \frac{d z_{0}}{d t}=-2 \alpha y_{0}-2 \epsilon z_{0}+\sigma_{2}^{2} .
\end{aligned}
$$

The eigenvalues of Eqs. (A3a)-(A3c) are $\lambda_{1}=-\lambda_{0}, \lambda_{2}=$ $-\lambda_{0}+\sqrt{\lambda_{0}^{2}-4 w_{o}^{2}}$, and $\lambda_{3}=-\lambda_{0}-\sqrt{\lambda_{0}^{2}-4 w_{o}^{2}}$. The solution converges to a steady state as the real parts of $\lambda_{1}, \lambda_{2}$, and $\lambda_{3}$ are all negative. The steady-state solution is

$$
\begin{aligned}
& x_{0}=\frac{\left(\alpha F+\epsilon \lambda_{0}+\epsilon^{2}\right) \sigma_{1}^{2}+F^{2} \sigma_{2}^{2}}{2\left(\lambda_{0}+\epsilon\right)\left(\alpha F+\epsilon \lambda_{0}\right)}, \\
& y_{0}=\frac{-\alpha \epsilon \sigma_{1}^{2}+F \lambda_{0} \sigma_{2}^{2}}{2\left(\lambda_{0}+\epsilon\right)\left(\alpha F+\epsilon \lambda_{0}\right)}, \\
& z_{0}=\frac{\alpha^{2} \sigma_{1}^{2}+\left(\alpha F+\lambda_{0}^{2}+\epsilon \lambda_{0}\right) \sigma_{2}^{2}}{2\left(\lambda_{0}+\epsilon\right)\left(\alpha F+\epsilon \lambda_{0}\right)} .
\end{aligned}
$$

The equations for the first order are

$$
\begin{aligned}
& \frac{d x_{1}}{d t}=-2 \lambda_{0} x_{1}+2 F y_{1}-2 x_{0} g(t) \\
& \frac{d y_{1}}{d t}=-\alpha x_{1}-\left(\lambda_{0}+\epsilon\right) y_{1}+F z_{1}-y_{0} g(t) \\
& \frac{d z_{1}}{d t}=-2 \alpha y_{1}-2 \epsilon z_{1}
\end{aligned}
$$

For the cyclostationary state, the zeroth-order solutions $\left(x_{0}, y_{0}\right.$, and $z_{0}$ ) are constant. As such, these zeroth-order solutions can be treated as constants in cyclostationary states. Equations (A5a)-(A5c) are then rewritten as

$$
\frac{d \mathbf{x}_{1}}{d t}=\mathbf{M x}+\mathbf{g}(t)
$$

where

$$
\mathbf{M}=\left(\begin{array}{ccc}
-2 \lambda_{0} & 2 F & 0 \\
-\alpha & -\left(\lambda_{0}+\epsilon\right) & F \\
0 & -2 \alpha & -2 \epsilon
\end{array}\right)
$$

and

$$
\mathbf{g}(t)=\left(\begin{array}{c}
-2 x_{0} g(t) \\
-y_{0} g(t) \\
0
\end{array}\right)
$$

We adopt the parameter variation method, a canonical method to solve nonhomogeneous linear ODEs. The solution is divided by $\mathbf{x}_{1}(t)=\mathbf{x}_{h}(t)+\mathbf{x}_{p}(t)$, where $\mathbf{x}_{h}(t)$ is the homogeneous solution that satisfies $d \mathbf{x}_{h} / d t=\mathbf{M} \mathbf{x}_{h}$, and $\mathbf{x}_{p}(t)$ is a particular solution. A particular solution is written as $\mathbf{x}_{p}=\mathbf{Y}(t)$ $\mathbf{u}(t)$, where $\mathbf{Y}(t)$ is the fundamental matrix of Eq. (A6). The problem is reduced to $d \mathbf{u} / d t=\mathbf{Y}^{-1}(t) \mathbf{g}(t)$. Hence, 

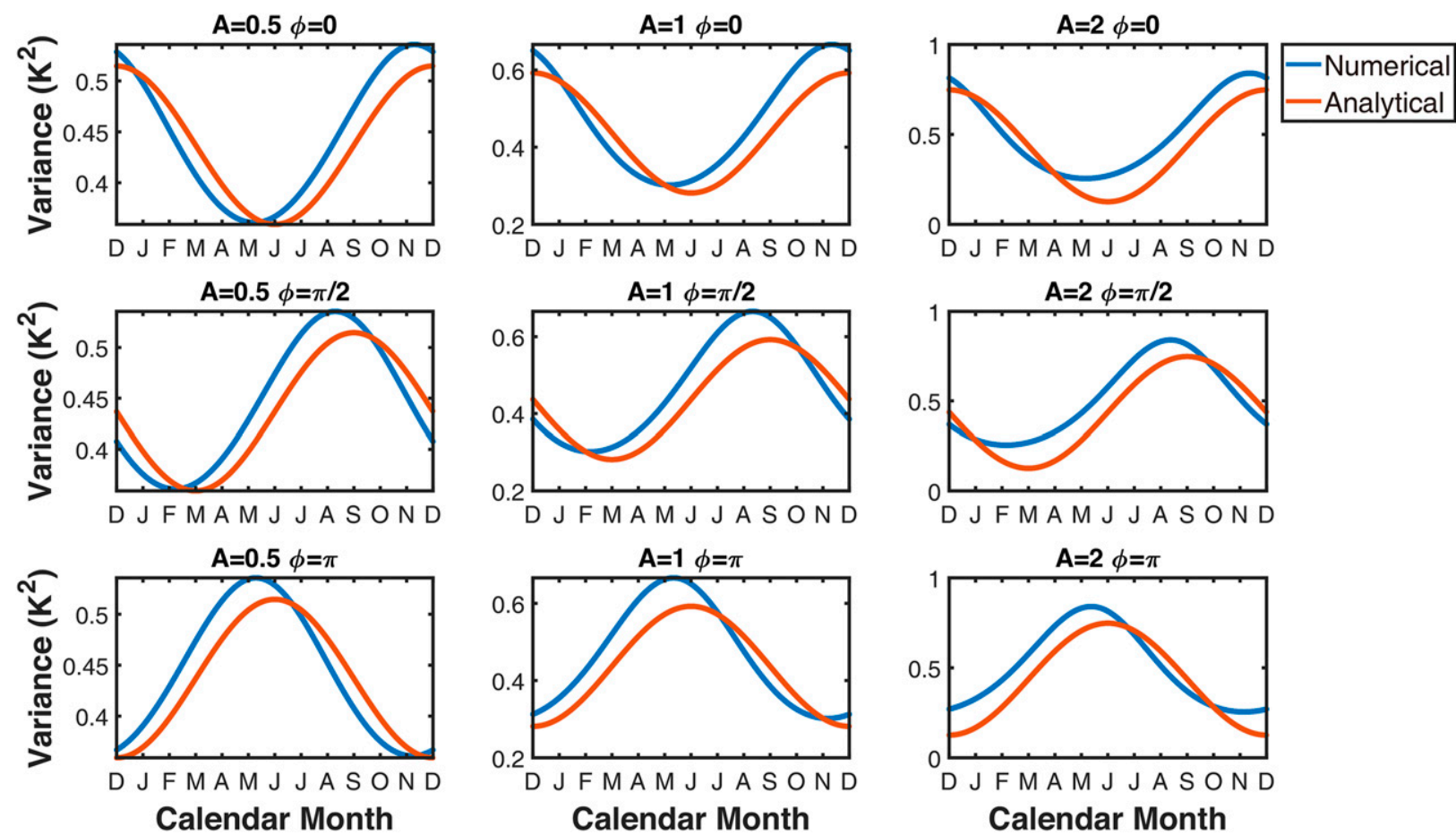

FIG. A1. Comparison between the numerical (blue line) and analytical (orange line) solutions for the seasonal variance of ENSO [Eq. (4)] under various parameter conditions for $f(t)=A \sin \left(w_{A} t+\phi\right)$. The numerical solution is obtained from the FPE [Eq. (2)]. The vertical and horizontal axes denote the variance (unit: $\mathrm{K}^{2}$ ) and calendar month, respectively.

$$
\mathbf{x}_{1}(t)=\mathbf{x}_{h}(t)+\mathbf{x}_{p}(t)=\mathbf{x}_{h}(t)+\mathbf{Y}(t) \int_{0}^{t} \mathbf{Y}^{-1}(\tau) \mathbf{g}(\tau) d \tau .
$$

For the cyclostationary state, $\mathbf{x}_{h}(t)$ becomes zero, as shown in the zeroth-order solution, because it is identical to the steadystate solution of Eqs. (A4a)-(A4c) with $\sigma_{1}^{2}=\sigma_{2}^{2}=0$. Hence, we only consider $\mathbf{x}_{p}(t)$ for the cyclostationary solution of $\mathbf{x}_{1}(t)$. Expanding Eq. (A7),

$$
\begin{aligned}
\mathbf{x}_{1}(t) & =\mathbf{Y}(t) \int_{0}^{t} \mathbf{Y}^{-1}(\tau) \mathbf{g}(\tau) d \tau \\
& =\mathbf{Y}(t)\left[\mathbf{Y}^{-1}(t) \mathbf{G}(t)-\mathbf{Y}^{-1}(0) \mathbf{G}(0)-\int_{0}^{t} \frac{d \mathbf{Y}^{-1}(\tau)}{d \tau} \mathbf{g}(\tau) d \tau\right] \\
& =\mathbf{G}(t)-\mathbf{Y}(t) \mathbf{Y}^{-1}(0) \mathbf{G}(0)-\mathbf{Y}(t) \int_{0}^{t} \frac{d \mathbf{Y}^{-1}(\tau)}{d \tau} \mathbf{g}(\tau) d \tau
\end{aligned}
$$

where $\mathbf{G}(t)=\int \mathbf{g}(t) d t$. Taking the limit $(t \rightarrow \infty)$ of Eq. (A8),

$$
\begin{aligned}
\lim _{t \rightarrow \infty} \mathbf{x}_{1}(t) & =\lim _{t \rightarrow \infty}\left[\mathbf{G}(t)-\mathbf{Y}(t) \mathbf{Y}^{-1}(0) \mathbf{G}(0)-\mathbf{Y}(t) \int_{0}^{t} \frac{d \mathbf{Y}^{-1}(\tau)}{d \tau} \mathbf{g}(\tau) d \tau\right] \\
& =\mathbf{G}(t)-\lim _{t \rightarrow \infty}\left[\mathbf{Y}(t) \int_{0}^{t} \frac{d \mathbf{Y}^{-1}(\tau)}{d \tau} \mathbf{g}(\tau) d \tau\right]
\end{aligned}
$$

as $\lim \mathbf{Y}(t)=0$. Note that $\lim \mathbf{Y}^{-1}(\tau)=\infty, d \mathbf{Y}^{-1}(\tau) / d \tau$ is written as follows [not explicitly shown for $\mathbf{Y}^{-1}(t)$ due to its complexity]:

$$
\frac{d \mathbf{Y}^{-1}(\tau)}{d \tau}=\mathbf{Y}^{-1}(\tau)\left(\begin{array}{ccc}
-\lambda_{1} & 0 & 0 \\
0 & -\lambda_{2} & 0 \\
0 & 0 & -\lambda_{3}
\end{array}\right)
$$

Therefore, $\left[d \mathbf{Y}^{-1}(\tau) / d \tau\right] \mathbf{g}(\tau)$ is calculated as

$$
\begin{aligned}
\frac{d \mathbf{Y}^{-1}(\tau)}{d \tau} \mathbf{g}(\tau) & =\mathbf{Y}^{-1}(\tau)\left(\begin{array}{ccc}
-\lambda_{1} & 0 & 0 \\
0 & -\lambda_{2} & 0 \\
0 & 0 & -\lambda_{3}
\end{array}\right)\left(\begin{array}{l}
g_{1}(\tau) \\
g_{2}(\tau) \\
g_{3}(\tau)
\end{array}\right) \\
& =-\mathbf{Y}^{-1}(\tau)\left(\begin{array}{c}
\lambda_{1} g_{1}(\tau) \\
\lambda_{2} g_{2}(\tau) \\
\lambda_{3} g_{3}(\tau)
\end{array}\right)
\end{aligned}
$$

Substituting Eqs. (A11) into (A9), and approximating the eigenvalue as zero $\left(\lambda_{1} \ll 1, \lambda_{2} \ll 1\right.$ and $\left.\lambda_{3} \ll 1\right)$, we obtain

$$
\lim _{t \rightarrow \infty} \mathbf{x}_{1}(t) \approx \mathbf{G}(t)=\left(\begin{array}{c}
-2 x_{0} \int g(t) d t \\
-y_{0} \int g(t) d t \\
0
\end{array}\right) .
$$

Finally, combining the zeroth- and first-order solutions, $\mathbf{x} \approx$ $\mathbf{x}_{0}+\epsilon \mathbf{x}_{1}$ gives 


$$
\begin{aligned}
\left(\begin{array}{l}
x \\
y \\
z
\end{array}\right) & \approx\left(\begin{array}{l}
x_{0} \\
y_{0} \\
z_{0}
\end{array}\right)+\epsilon\left(\begin{array}{c}
-2 x_{0} \int g(t) d t . \\
-y_{0} \int g(t) d t . \\
0
\end{array}\right) \\
& =\left(\begin{array}{c}
x_{0}\left[1-2 \int \epsilon g(t) d t\right] \\
y_{0}\left[1-\int \epsilon g(t) d t\right] \\
z_{0}
\end{array}\right)=\left(\begin{array}{c}
x_{0}\left[1-2 \lambda_{0} \int f(t) d t\right] \\
y_{0}\left[1-\lambda_{0} \int f(t) d t\right] \\
z_{0}
\end{array}\right) .
\end{aligned}
$$

Substituting Eq. (A4a) into Eq. (A13), and the variance of SSTA $(x)$ becomes

$$
\sigma_{T}^{2}(t) \approx \frac{\left(\alpha F+\epsilon \lambda_{0}+\epsilon^{2}\right) \sigma_{1}^{2}+F^{2} \sigma_{2}^{2}}{2\left(\lambda_{0}+\epsilon\right)\left(\alpha F+\epsilon \lambda_{0}\right)}\left[1-2 \lambda_{0} \int f(t) d t\right] .
$$

To validate Eq. (A14), the solution is compared with the numerical solution obtained from FPE [Eq. (2)] under various $A$ and $\phi$ conditions (Fig. A1). The FPE is time-integrated for 10 years, and the last year is taken to obtain seasonal variance in the cyclostationary state. The analytical solution matches well with the numerical solution in terms of its overall range and maxima/minima timing. This indicates that the adopted approximations are quite reasonable, but it has some underestimations and a peak delay of $\sim 0.5$ months.

\section{APPENDIX B}

\section{Derivation of the Critical Point for the Seasonal Gap}

In this section, we derive the critical point for the seasonal gap of ENSO. The SROM [Eqs. (1a) and (1b)] excluding noise $\left(\sigma_{1}=\sigma_{2}=0\right)$ is converted to a second-order ODE of $T$ :

$$
\frac{d^{2} T}{d t^{2}}+\beta(t) \frac{d T}{d t}+w^{2}(t) T=0,
$$

where $\beta(t)=\lambda_{0}+\epsilon+\lambda_{0} f(t)$ and $w^{2}(t)=\lambda_{0} \epsilon+\alpha F+\lambda_{0} \epsilon f(t)+$ $\lambda_{0}(d f / d t)$. The converted second-order ODE of $T$ [Eq. (B1)] is equivalent to a parametric oscillator, which is a damped harmonic oscillator with time-varying physical properties (see https://en.wikipedia.org/wiki/Parametric_oscillator). Introducing a new variable,

$$
q(t)=T(t) \exp \left[\frac{1}{2} \int \beta(t) d t\right] .
$$

Substituting Eqs. (B2) into (B1),

$$
\frac{d^{2} q}{d t^{2}}+\Omega^{2}(t) q=0
$$

where $\Omega^{2}(t)=w^{2}(t)-(1 / 2)(d \beta / d t)-(1 / 4) \beta(t)^{2}$. The peak of $T$ satisfies $d T / d t=0$ by definition, and such a condition is expressed as $q(t)$ :

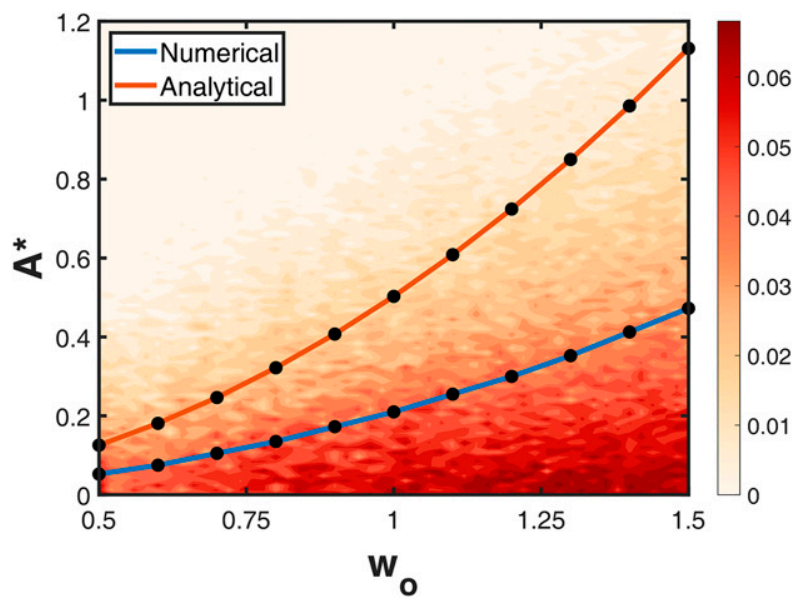

FIG. B1. Comparison between the numerical (blue line) and analytical (orange line) solutions [Eq. (8)] for the critical point of the seasonal gap $\left(A^{*}\right)$ under various ENSO linear frequency conditions $\left(w_{o}\right)$. Here, $w_{o}$ is normalized for the default linear parameters outlined in section 2. The red color denotes the minimum PDF value of the peak calendar month for the given $A$ and $w_{o}$ under noise forcing. The lower (higher) value signifies proximity to the strong (weak) phase locking regime. Equations (1a) and (1b) including noise are integrated for 50000 years and the peak calendar months are obtained from an El Niño (La Niña) event criteria outlined in the main text. Note the $y$ axis for the contour is $A$ rather than $A^{*}$, but it is plotted together with numerical/analytical $A^{*}$ for a direct comparison.

$$
\frac{d q}{d t}=\frac{1}{2} \beta(t) q
$$

Defining the new function,

$$
g(t)=\frac{2 r(t)}{\lambda_{0}}-\left(1+\frac{\epsilon}{\lambda_{0}}\right)-f(t),
$$

where $r=(1 / q)(d q / d t)$. Rewriting Eq. (B4) for $g(t)$ yields

$$
g(t)=0
$$

The seasonal gap bifurcation is identical to the double-peak bifurcation by increasing $A$ (see Fig. 5). Therefore, a critical point for the seasonal gap is identified by a single-to-double solution bifurcation of Eq. (B6) by $A$. The number of solutions for Eq. (B6) is determined by the shape of $g(t)$; a nonpeak (double extrema) shaped $g(t)$ yields a single (multiple) solution. The peak of the solution can be found by

$$
\frac{d g}{d t}=\frac{2}{\lambda_{0}} \frac{d r}{d t}-\frac{d f}{d t}=0 .
$$

Here, $d r / d t=(1 / q)\left(d^{2} q / d t^{2}\right)-\left(1 / q^{2}\right)(d q / d t)^{2}=-\Omega^{2}-r^{2}$, and

$$
\frac{2\left(\Omega^{2}+r^{2}\right)}{\lambda_{0}}+\frac{d f}{d t}=0 .
$$

Assuming $f(t)$ is very small $[f(t) \approx 0]$, we adopt the approximation $r^{2} \approx \Omega^{2}$. Applying the approximation in Eq. (B8), 


$$
\frac{4 \Omega^{2}}{\lambda_{0}}+\frac{d f}{d t}=0
$$

where $\Omega^{2}=\alpha F-(1 / 4)\left(\lambda_{0}-o\right)^{2}+\left(\lambda_{0} / 2\right)(d f / d t)-(1 / 2) \lambda_{0}\left(\lambda_{0}-\right.$ o) $f(t)-(1 / 4) \lambda_{0}^{2} f(t)^{2}$. The time-varying parameter $\Omega^{2}$ includes annual $[f(t)$ and $d f / d t]$ and semiannual cycle components $\left[f(t)^{2}\right]$. Ignoring the semiannual cycle component for $\Omega^{2}$ :

$$
\Omega^{2} \approx w_{o}^{2}+\frac{\lambda_{0}}{2} \frac{d f}{d t}-\frac{1}{2} \lambda_{0}\left(\lambda_{0}-\epsilon\right) f(t),
$$

where $w_{o}^{2}=\alpha F-(1 / 4)\left(\lambda_{0}-\epsilon\right)^{2}$.

Substituting Eqs. (B10) into (B9),

$$
\frac{1}{2} \lambda_{0}\left(\lambda_{0}-\epsilon\right) f(t)-\frac{3}{4} \lambda_{0} \frac{d f}{d t}=w_{o}^{2} .
$$

For $f(t)=A \sin \left(w_{a} t+\phi\right)$, Eq. (B10) then becomes

$$
A \sin \left(w_{a} t+\varphi\right)=\frac{w_{o}^{2}}{\lambda_{0} \sqrt{\frac{9}{16} w_{a}^{2}+\frac{1}{4}\left(\lambda_{0}-\epsilon\right)^{2}}},
$$

where $\tan \varphi=-(3 / 2) 1 /\left(\lambda_{0}-\epsilon\right)$. For Eq. (B11) to have multiple solutions, $A$ should be larger than the right-hand side of the equation. Therefore, the critical condition for the seasonal gap $\left(A^{*}\right)$ is

$$
A^{*} \approx \frac{w_{o}^{2}}{\lambda_{0} \sqrt{\frac{9}{16} w_{a}^{2}+\frac{1}{4}\left(\lambda_{0}-\epsilon\right)^{2}}} .
$$

To validate Eq. (B13), a comparison between the approximated analytical and numerical solutions is carried out under various ENSO intrinsic frequencies $\left(w_{o}\right)$ (Fig. B1). To directly compare the effect of $w_{o}$, we adopt the approximated version of the linear SROM by modifying the default parameter settings as $F=w_{o}$, $\alpha=w_{o}$, and $\epsilon=0$, where $w_{o}=0.14$. These results are shown in Fig. B1.

\section{REFERENCES}

Alexander, M. A. I. Bladé, M. Newman, J. R. Lanzante, N. Lau, and J. D. Scott, 2002: The atmospheric bridge: The influence of ENSO teleconnections on air-sea interaction over the global oceans. J. Climate, 15, 2205-2231, https://doi.org/10.1175/15200442(2002)015<2205:TABTIO>2.0.CO;2.

An, S.-I., 2008: Interannual variations of the tropical ocean instability wave and ENSO. J. Climate, 21, 3680-3686, https:// doi.org/10.1175/2008JCLI1701.1.

— and La Niña mature phases to boreal winter. J. Climate, 14, 2164-2176, https://doi.org/10.1175/1520-0442(2001)014<2164: MOLOTE $>2.0 . \mathrm{CO} ; 2$.

- , and F.-F. Jin, 2004: Nonlinearity and asymmetry of ENSO. J. Climate, 17, 2399-2412, https://doi.org/10.1175/1520-0442(2004) 017<2399:NAAOE $>2.0 . \mathrm{CO} ; 2$.

—, and —, 2011: Linear solutions for the frequency and amplitude modulation of ENSO by the annual cycle. Tellus, $\mathbf{6 3 A}$, 238-243, https://doi.org/10.1111/j.1600-0870.2010.00482.x.

—, S.-K. Kim, and A. Timmermann, 2020: Fokker-Planck dynamics of the El Niño-Southern Oscillation. Sci. Rep., 10, 16282, https://doi.org/10.1038/s41598-020-73449-7.
Battisti, D. S., and A. C. Hirst, 1989: Interannual variability in a tropical atmosphere-ocean model: Influence of the basic state, ocean geometry, and nonlinearity. J. Atmos. Sci., 46, 1687-1712, https://doi.org/10.1175/1520-0469(1989)046<1687: IVIATA $>2.0 . \mathrm{CO} ; 2$.

Behringer, D. W., M. Ji, and A. Leetmaa, 1998: An improved coupled model for ENSO prediction and implications for ocean initialization. Part I: The ocean data assimilation system. Mon. Wea. Rev., 126, 1013-1021, https://doi.org/10.1175/ 1520-0493(1998)126<1013:AICMFE > 2.0.CO;2.

Burgers, G., F.-F. Jin, and G. J. van Oldenborgh, 2005: The simplest ENSO recharge oscillator. Geophys. Res. Lett., 32, L13706, https://doi.org/10.1029/2005GL022951.

Chen, H. C., and F.-F. Jin, 2020: Fundamental behavior of ENSO phase locking. J. Climate, 33, 1953-1968, https://doi.org/ 10.1175/JCLI-D-19-0264.1.

Cole, J. E., J. T. Overpeck, and E. R. Cook, 2002: Multiyear La Niña events and persistent drought in the contiguous United States. Geophys. Res. Lett., 29, 1647, https://doi.org/10.1029/ $2001 G L 013561$.

Dommenget, D., and Y. Yu, 2016: The seasonally changing cloud feedbacks contribution to the ENSO seasonal phase-locking. Climate Dyn., 47, 3661-3672, https://doi.org/10.1007/s00382-016-3034-6.

Galanti, E., and E. Tziperman, 2000: ENSO's phase locking to the seasonal cycle in the fast-SST, fast-wave, and mixed-mode regimes. J. Atmos. Sci., 57, 2936-2950, https://doi.org/10.1175/ 1520-0469(2000)057<2936:ESPLTT>2.0.CO;2.

Giese, B. S., and S. Ray, 2011: El Niño variability in simple ocean data assimilation (SODA), 1871-2008. J. Geophys. Res., 116, C02024, https://doi.org/10.1029/2010JC006695.

Hayashi, M., and F.-F. Jin, 2017: Subsurface nonlinear dynamical heating and ENSO asymmetry. Geophys. Res. Lett., 44, 12 427-12 435, https://doi.org/10.1002/2017GL075771.

Hirst, A. C., 1986: Unstable and damped equatorial modes in simple coupled ocean-atmosphere models. J. Atmos. Sci., 43, 606-632, https://doi.org/10.1175/1520-0469(1986)043<0606: $\mathrm{UADEMI}>2.0 . \mathrm{CO} ; 2$.

Huang, B., and Coauthors, 2017: Extended Reconstructed Sea Surface Temperature, version 5 (ERSSTv5): Upgrades, validations, and intercomparisons. J. Climate, 30, 8179-8205, https://doi.org/10.1175/JCLI-D-16-0836.1.

Jin, F.-F., 1997: An equatorial ocean recharge paradigm for ENSO. Part I: Conceptual model. J. Atmos. Sci., 54, 811-829, https://doi.org/10.1175/1520-0469(1997)054<0811: AEORPF $>2.0 . \mathrm{CO} ; 2$.

— J. D. Neelin, and M. Ghil, 1994: El Niño on the devil's staircase: Annual subharmonic steps to chaos. Science, 264, 70-72, https://doi.org/10.1126/science.264.5155.70.

Jin, Y., Z. Liu, Z. Lu, and C. He, 2019: Seasonal cycle of background in the tropical Pacific as a cause of ENSO spring persistence barrier. Geophys. Res. Lett., 46, 13 371-13 378, https:// doi.org/10.1029/2019GL085205.

Kim, S.-K., and S.-I. An, 2020: Untangling El Niño-La Niña asymmetries using a nonlinear coupled dynamic index. Geophys. Res. Lett., 47, e2019GL085881, https://doi.org/10.1029/2019GL085881.

Levine, A. F., and F.-F. Jin, 2010: Noise-induced instability in the ENSO recharge oscillator. J. Atmos. Sci., 67, 529-542, https:// doi.org/10.1175/2009JAS3213.1.

Li, T., 1997: Phase transition of the El Niño-Southern Oscillation: A stationary SST mode. J. Atmos. Sci., 54, 2872-2887, https:// doi.org/10.1175/1520-0469(1997)054<2872:PTOTEN>2.0.CO;2. , and P.-C. Hsu, 2017: Fundamentals of Tropical Climate Dynamics. Springer, 229 pp. 
Okumura, Y. M., and C. Deser, 2010: Asymmetry in the duration of El Niño and La Niña. J. Climate, 23, 5826-5843, https://doi.org/ 10.1175/2010JCLI3592.1.

Park, J.-H., S.-I. An, J.-S. Kug, Y.-M. Yang, T. Li, and H.-S. Jo, 2021: Mid-latitude leading double-dip La Niña. Int. J. Climatol., 41, E1353-E1370, https://doi.org/10.1002/joc.6772.

Philander, S. G. H., 1983: El Niño Southern Oscillation phenomena. Nature, 302, 295-301, https://doi.org/10.1038/302295a0.

— , T. Yamagata, and R. C. Pacanowski, 1984: Unstable air-sea interactions in the tropics. J. Atmos. Sci., 41, 604-613, https:// doi.org/10.1175/1520-0469(1984)041<0604:UASIIT>2.0.CO;2.

Risken, H., 1989: The Fokker-Planck Equation: Methods of Solution and Applications. Springer, $485 \mathrm{pp}$.

Särkkä, S., and A. Solin, 2019: Applied Stochastic Differential Equations. Cambridge University Press, 316 pp.

Stein, K., N. Schneider, A. Timmermann, and F.-F. Jin, 2010: Seasonal synchronization of ENSO events in a linear stochastic model. J. Climate, 23, 5629-5643, https://doi.org/10.1175/ 2010JCLI3292.1.

— A. Timmermann, N. Schneider, F.-F. Jin, and M. F. Stuecker, 2014: ENSO seasonal synchronization theory. J. Climate, 27, 5285-5310, https://doi.org/10.1175/JCLI-D-13-00525.1.
Takahashi, K., and B. Dewitte, 2016: Strong and moderate nonlinear El Niño regimes. Climate Dyn., 46, 1627-1645, https:// doi.org/10.1007/s00382-015-2665-3.

Timmermann, A., and Coauthors, 2018: El Niño-Southern Oscillation complexity. Nature, 559, 535-545, https://doi.org/10.1038/s41586018-0252-6.

Tziperman, E., L. Stone, M. A. Cane, and H. Jarosh, 1994: El Niño chaos: Overlapping of resonances between the seasonal cycle and the Pacific ocean-atmosphere oscillator. Science, 264, 7274, https://doi.org/10.1126/science.264.5155.72.

_ S. E. Zebiak, and M. A. Cane, 1997: Mechanisms of seasonalENSO interaction. J. Atmos. Sci., 54, 61-71, https://doi.org/ 10.1175/1520-0469(1997)054<0061:MOSEI > 2.0.CO;2.

Xie, S.-P., K. Hu, J. Hafner, H. Tokinaga, Y. Du, G. Huang, and T. Sampe, 2009: Indian Ocean capacitor effect on Indo-western Pacific climate during the summer following El Niño. J. Climate, 22, 730-747, https://doi.org/10.1175/2008JCLI2544.1.

Xue, A., F.-F. Jin, W. Zhang, J. Boucharel, S. Zhao, and X. Yuan, 2020: Delineating the seasonally modulated nonlinear feedback onto ENSO from tropical instability waves. Geophys. Res. Lett., 47, e2019GL085863, https://doi.org/10.1029/ 2019GL085863. 